\title{
Studies of parton distributions at a neutrino factory
}

\author{
S. Kumano* \\ Department of Physics \\ Saga University \\ Saga, 840-8502, Japan
}

Talk given at the Third International Workshop on

Neutrino Factories based on Muon Storage Rings

(NuFACT'01)

May 24-30, 2001, Tsukuba, Japan

(talk on May 28, 2001)

* Email: kumanos@cc.saga-u.ac.jp. Information on his research is available at http://hs.phys.saga-u.ac.jp.

to be published in proceedings 


\title{
Studies of parton distributions at a neutrino factory
}

\author{
S. Kumano \\ Department of Physics, Saga University \\ Honjo-1, Saga, 840-8502, Japan
}

\begin{abstract}
The determination of parton distribution functions in the nucleon and nuclei is important for obtaining precise hadron-reaction cross sections, from which any new exotic signature could be found. We show that a future neutrino factory could provide important information on the parton distributions. First, a recent effort concerning the parametrization of nuclear parton distributions is explained. It suggests that the factory should be important for determining unknown behavior of valence-quark distributions in nuclei at small $x$. Second, the facility could be used for understanding nucleon spin structure and isospin violation in the parton distributions.
\end{abstract}

\section{Nuclear parton distributions}

Unpolarized parton distribution functions are now well known from very small $x$ to relatively large $x$. However, polarized distributions and nuclear parton distributions are not precisely determined at this stage because of the lack of a variety of data. In the recent years, several parametrizations of polarized distributions have been proposed. It was, however, unfortunate that there was no available $\chi^{2}$ analysis for nuclear distributions until recently. An initial effort of such a nuclear parametrization is discussed in Ref. [1]. The nuclear parton distributions are provided at $Q_{0}^{2}=1 \mathrm{GeV}^{2}$ as $f_{i}^{A}\left(x, Q_{0}^{2}\right)=w_{i}(x, A, Z) f_{i}\left(x, Q_{0}^{2}\right)$ with $i=u_{v}, d_{v}, \bar{q}$, or $g$. Here, $f_{i}\left(x, Q_{0}^{2}\right)$ is a distribution in the nucleon, and $w_{i}(x, A, Z)$ is a weight function which takes into account nuclear modification. It is defined with parameters $a_{i}, b_{i}, c_{i}, d_{i}$, and $\beta_{i}$ :

$$
w_{i}(x, A, Z)=1+\left(1-\frac{1}{A^{1 / 3}}\right) \frac{a_{i}(A, Z)+b_{i} x+c_{i} x^{2}+d_{i} x^{3}}{(1-x)^{\beta_{i}}} .
$$

Then, a $\chi^{2}$ analysis was performed by including electron and muon deep inelastic experimental data. We call this analysis a "cubic fit", and that without the $d_{i} x^{3}$ term is called a "quadratic fit". The details can be found in Ref. [1]. 
The obtained weight functions for the calcium nucleus are shown in Fig.1, where the solid curves are cubicfit results and the dashed curves are quadratic ones. A determination of the valence-quark distribution at small $x$ is not possible from only the electron and muon data. Model predictions are also confusing in the

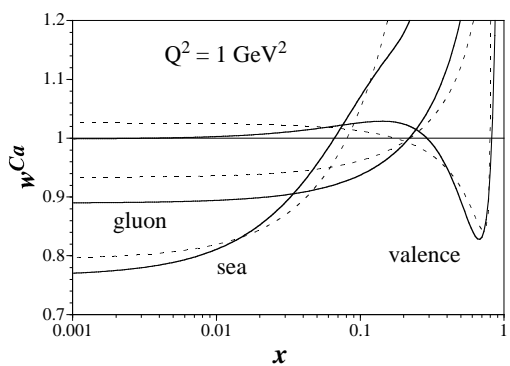

Fig. 1. Obtained weight functions. sense that some models predict shadowing for the valence quark, and the others predict antishadowing [2]. However, the parity-violating structure function $\left(F_{3}\right)$ in the neutrino interaction provides an important clue in determining the valence distributions at small $x$ :

$$
\frac{1}{2}\left[F_{3}^{\nu(p+n) / 2}+F_{3}^{\bar{\nu}(p+n) / 2}\right]=u_{v}+d_{v}+(s-\bar{s})+(c-\bar{c}) .
$$

Because $s-\bar{s}$ and $c-\bar{c}$ are expected to be small, neutrino-factory experiments provide accurate determination of the valence distributions in nuclei.

There are available neutrino deep inelastic data, which are indeed used in the nucleon parametrization. Although they play an important role in determining the valence-quark distribution in the "nucleon", the used target has been iron. Nuclear modification is not properly taken into account in the parametrization. This fact suggests that the valence distributions in the nucleon should be re-investigated with the nuclear modification. If a neutrino factory becomes available, it should be possible to take accurate deuteron data, namely the ratios $F_{3}^{A} / F_{3}^{D}$. Then, the neutrino data can be accommodated into the aforementioned nuclear parametrization.

\section{Selected topics}

Polarized valence-quark distributions

We have also investigated optimum polarized parton distributions in the nucleon [3] by analyzing polarized electron and muon deep inelastic data. The situation is similar to the nuclear case in the previous section in the sense that each parton distributions cannot be well determined without accurate hadron collider data. In the same way as $F_{3}$, a parity-violating structure function $\left(g_{3}\right)$ exists. By combining proton and neutron structure functions, we obtain in the leading order of $\alpha_{s}[4]$ :

$$
\begin{aligned}
g_{3}^{\nu p}+g_{3}^{\bar{\nu} p} & =-\left(\Delta u_{v}+\Delta d_{v}\right)-(\Delta s-\Delta \bar{s})-(\Delta c-\Delta \bar{c}), \\
g_{3}^{\bar{\nu}(p+n) / 2}-g_{3}^{\nu(p+n) / 2} & =(\Delta s+\Delta \bar{s})-(\Delta c+\Delta \bar{c}) .
\end{aligned}
$$

These equations suggest that the neutrino facility is valuable not only for the polarized valence-quark distributions, but also for the antiquark distributions. 
$\underline{\text { Flavor asymmetry in light antiquark distributions }}$

The $\bar{u} / \bar{d}$ asymmetry in the nucleon is now well established by the NMC's Gottfried-sum-rule (GSR) violation and succeeding Drell-Yan experiments. It is nonetheless important to confirm it in a totally different process, such as the neutrino reaction. Furthermore, it should be important to find unknown polarized flavor asymmetry. These distributions are expressed in terms of the neutrino structure functions as [5]

$$
\begin{aligned}
\bar{u}-\bar{d} & =\frac{1}{4}\left[F_{2}^{\nu p} / x-F_{3}^{\nu p}\right]-\frac{1}{4}\left[F_{2}^{\nu n} / x-F_{3}^{\nu n}\right], \\
\Delta \bar{u}-\Delta \bar{d} & =\frac{1}{2}\left[g_{1}^{\nu p}+g_{3}^{\nu p}\right]-\frac{1}{2}\left[g_{1}^{\nu n}+g_{3}^{\nu n}\right] .
\end{aligned}
$$

Isospin symmetry

The isospin symmetry in the parton distributions is taken for granted. In fact, it is generally believed that the isospin-violation is of the order of a few $\%$ $(\sim \alpha)$. By combining the $F_{2}$ structure functions, we could test this "common sense" at the neutrino factory in connection with the GSR violation [5]

$$
\begin{aligned}
& \int \frac{d x}{4 x}\left[F_{2}^{(\nu+\bar{\nu}) p}-F_{2}^{(\nu+\bar{\nu}) n}\right]=\int d x\left[(\bar{u}+\bar{d}+\bar{s}+\bar{c})_{p}-(\bar{u}+\bar{d}+\bar{s}+\bar{c})_{n}\right] \\
&=0 \quad \text { if isospin symmetry is satisfied, } \\
& \neq 0 \quad \text { if the GSR violation is partly due to } \\
& \text { isospin symmetry violation. }
\end{aligned}
$$

\section{Summary}

We learned that a future neutrino factory could provide important information for the parton distributions in the nucleon and nuclei. Because the distributions are essential for calculating precise hadron cross sections, our studies are important for finding a new signature beyond the current theoretical framework.

\section{Acknowledgments}

S.K. was supported by a Grant-in-Aid for Scientific Research from the Japanese Ministry of Education, Culture, Sports, Science, and Technology. He also thanks RIKEN for supporting his participation in this conference.

\section{References}

[1] M. Hirai, S. Kumano, and M. Miyama, Phys. Rev. D64 (2001) 034003. See http://hs.phys.saga-u.ac.jp/nuclp.html for a nuclear parton distribution library.

[2] R. Kobayashi, S. Kumano, and M. Miyama, Phys. Lett. B354 (1995) 465.

[3] Y. Goto et. al. (AAC), Phys. Rev. D62 (2000) 034017. The AAC library is available at http://spin.riken.bnl.gov/aad.

[4] B. Lampe and E. Reya, Phys. Rep. 332 (2000) 1.

[5] S. Kumano, Phys. Rep. 303 (1998) 183. 